\title{
Meta-analysis of transcriptome data clarified hormonal regulation of cold stress response in Arabidopsis thaliana L.
}

Nadezda Omelyanchuk Institute of Cytology and Genetics SB RAS

Novosibirsk, Russia nadya@bionet.nsc.ru

\author{
Yana Sizentsova \\ Institute of Cytology and \\ Genetics SB RAS \\ Novosibirsk, Russia \\ sizentsova.yans@gmail.ru
}

\author{
Victoria Mironova \\ Institute of Cytology and \\ Genetics SB RAS, \\ Novosibirsk State University, \\ Novosibirsk, Russia \\ kviki@bionet.nsc.ru
}

\begin{abstract}
Low positive temperatures not only have damaging effects on plants in various climate from tropics to temperate zones but also provide acclimation to freezing for many plant species. It is why cold stress response is an actual topic for a wide range of experiments, including whole-genome studies. The results of these experiments are diverse and, in many cases, even contradictory. A meta-analysis of wholegenome data is a useful approach to distinguish between the robust effects of low temperature itself and the influence of particular experimental conditions. Here we applied metaanalysis to transcriptome data sets on cold stress response in the model plant species Arabidopsis thaliana, to find out changes in plant hormone metabolism and signaling accompanying this process. We identified that cold stress dramatically changes the plant hormonal status. Here we discuss the dynamics of the changes for each hormone.
\end{abstract}

Keywords - cold stress, phytohormones, transcriptome, metaanalysis, Arabidopsis thaliana

\section{Introduction}

Cold stress greatly limits geographic distribution of agricultural crops. Plants from temperate zones have evolved a number of mechanisms to cope with chilling and freezing stresses, including delay in germination and flowering timing, inhibition of growth etc. Plant hormones are key intermediators between changes in environment and plant developmental reaction to them. Nevertheless, our knowledge on hormonal input into reaction to low positive temperature is enough patchy [1]. For instance, the role of auxin, the major regulator of plant growth and development, has not been studied in response to cold, although cold has been shown to increase expression of auxin-regulated genes and increase auxin accumulation. Brassinosteroids involvement in plant growth and development during cold stress in also unclear. One of the main reasons for this are intensive cross-talks between hormones themselves and their involvement in reactions to other environmental stimuli. The meta-analysis of many transcriptome experiments (microarrays and RNA-seqs) helps to exclude the differences triggered by variations in experimental design and to highlight the most robust pattern for involvement of hormones in cold response.

\section{materials and Methods}

We compiled a list of publically available datasets on cold stress response in Arabidopsis thaliana (40 datasets in total). Microarray raw data was processed using RMA algorithm implemented in the Bioconductor affy [2] and limma [3] packages. RNA-Seq data pre-processing and TMM normalization was done using edgeR package [4]. As a result, we compiled two matrixes for all 40 datasets with information about DEGs: (1) with $\log 2 \mathrm{FC}$ and (2) False discovery rates
(FDR). To compare the datasets we applied PCA and hierarchical cluster analysis using stats $\mathrm{R}$ package [5].

Then we selected the genes that robustly activated or inhibited in response to cold stress over a number of datasets (the threshold was calculated by binomial test).

Functional enrichment analysis was done using DAVID v6.8 [6].

\section{Results}

Firstly, we performed functional annotation of the robustly up or down-regulated genes in either early, late or very late response. Among the overrepresented GO categories we paid especial attention to those associated with hormonal metabolism and signaling. The results are summarized on the figure 1. Functional annotation showed that pathways of almost all hormones were activated at early stage of cold stress except auxin and citokinin pathways. Citokinin metabolism and signaling were only activated at very late stages of cold stress, whereas auxin signaling was both up- and downregulated.

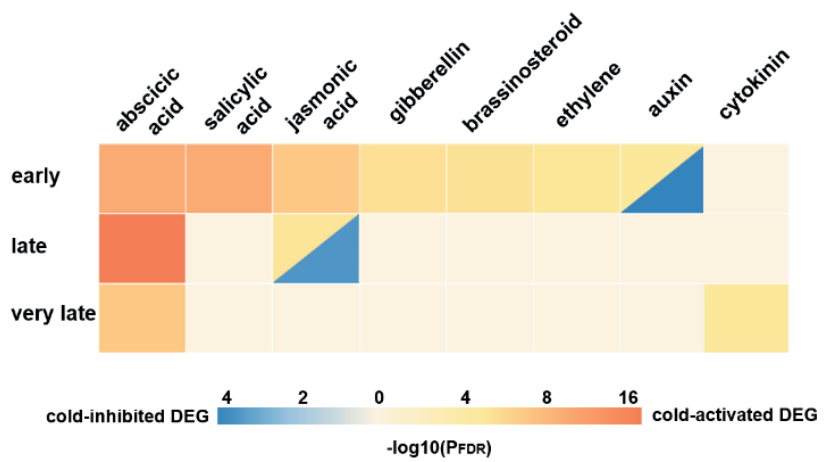

Fig. 1. Functional annotation results for early, late and very late cold response in Arabidopsis thaliana. Only GO terms associated with hormonal control are shown.

As Gene Ontology is rather incomplete and redundant, we compiled a list of genes mediating synthesis, degradation, conjugation, transport and synthesis of main plant hormones to better understand the gene expression changes in hormone pathways. We analyzed which of them robustly regulated by cold in early, late or very late response. The analytical results are summed up below.

In the early phase of response to the cold stress, abscisic acid, brassinosteroid and ethylene signaling increases whereas jasmonic acid and auxin signaling, in opposite, decreases. In the late phase fine-tuning of these signaling occurred, which means that each of these pathways has at least one upregulated component along with at least one suppressed. For most of 
hormones, fine-tuning of signaling continues in the very late phase, where solely auxin and gibberellin signals increase and decrease, respectively. Cytokinin and salicylic acid signaling starting correspondingly from the early and late phases of cold response have only "fine-tuning" mode of regulation. Similar to salicylic acid, gibberellin signaling begins from the late phase.

Many of these changes in signaling may come from the changes in biosynthesis, degradation and/or conjugation and distribution of hormones. First, in the early phase, cold activates biosynthesis of abscisic and jasmonic acids, ethylene, gibberellin and auxin, and then in the late phase cytokinin biosynthesis is increased. While at the early phase, only activation of hormone biosynthesis takes place at later stages suppression (brassinosteroids) and fine-tuning (abscisic, jasmonic and salicylic acids and gibberellin) are switching on. Ethylene biosynthesis was activated, fine-tuned and suppressed at the early, late and very late phases, respectively.

Both degradation and conjugation are processes leading to decreasing of the hormone level. Degradation and/or conjugation starts in the early phase for abscisic acid, brassinosteroids and cytokinin, and later is inhibited for brassinosteroids and fine-tuned for abscisic and jasmonic acids, cytokinin and auxin. At the early phase of cold response, changes in metabolism occur for all hormones under study except salicylic acid, which react to cold later.
Thus, with some exceptions, cold activates hormone metabolism and/or signaling at the early phase of cold response fine-tuning them at the later stages possibly mainly due to intensive crosstalk between signaling and metabolic pathways of various hormones.

\section{Acknowledgments}

This work was supported by Russian Science Foundation 18-74-10008.

\section{References}

[1] M. Eremina, W. Rozhon, and B. Poppenberger, "Hormonal control of cold stress responses in plants", (in eng), Cell Mol Life Sci, vol. 73, no. 4, pp. 797-810, Feb 2016.

[2] L. Gautier, L. Cope, B. M. Bolstad, and R. A. Irizarry, "Affy-analysis of Affymetrix GeneChip data at the probe level", Bioinformatics, vol. 20, no. 3, pp. 307-15, Feb 2004.

[3] M. E. Ritchie et al., "Limma powers differential expression analyses for RNA-sequencing and microarray studies", Nucleic Acids Res, vol. 43, no. 7, p. e47, Apr 2015.

[4] M. D. Robinson, D. J. McCarthy, and G. K. Smyth, "EdgeR: a Bioconductor package for differential expression analysis of digital gene expression data", Bioinformatics, vol. 26, no. 1, pp. 139-40, Jan 2010.

[5] R Core Team, "R: A language and environment for statistical computing", R Foundation for Statistical, 2019.

[6] D. W. Huang, B. T. Sherman, and R. A. Lempicki, "Bioinformatics enrichment tools: paths toward the comprehensive functional analysis of large gene lists", Nucleic Acids Res, vol. 37, no. 1, pp. 1-13, Jan 2009 . 